\title{
ANÁLISE DO ESTUDO DE NÃO-UNIFORMIDADE DE UM SIMULADOR SOLAR DE GRANDE ÁREA UTILIZADO PARA CARATERIZAÇÃO DE PAINÉIS SOLARES
}

Larissa de Oliveira Rocha ${ }^{1}$; Erika Dourado Cardeal $^{1}$; José Alejandro Moreno Alfonzo ${ }^{123}$; Tiago Franca Paes ${ }^{12}$; Marcus Vinicius Santos da Silva1; luri Muniz Pepe ${ }^{23}$; Denis Gilbert Francis ${ }^{1}$.

1 Laboratório de Certificação de Componentes de Sistemas de Energia Solar Fotovoltaica IF-UFBA; Tecnocentro, Salvador/BA; erika_dourado14@outlook.com

2 Laboratório de Propriedades Óticas IF-UFBA; Ondina, Salvador/BA

${ }^{3}$ Programa de Pós-graduação em Mecatrônica UFBA; Ondina, Salvador/BA

Resumo: Este trabalho apresenta os resultados do estudo de Não-Uniformidade em um Simulador Solar desenvolvido no Laboratório de Certificação de Sistemas de Energia Solar Fotovoltaica. Estes resultados são utilizados para o cálculo do erro determinante na obtenção da classificação em conformidade com a norma IEC 609049. O procedimento e quantidade de pontos para este tipo de análise é definido pela mesma norma, o que, segundo nossos resultados, traria erros na estimativa da classificação nos primeiros procedimentos de calibração. Mediante diversos mapas com quantidade de pontos de medida crescentes de 2 a 1620, observou-se em uma análise de convergência que é necessário aumentar o número de medidas até achar um valor do erro mais próximo ao real.

Palavras-Chave: Simulador Solar, Não-Uniformidade, Normas.

\section{REVIEW OF NON-UNIFORMITY STUDY OF A LARGE SCALE SOLAR SIMULATOR USED FOR SOLAR PANEL CHARACTERIZATION}

\begin{abstract}
This paper presents the results of the non-uniformity study of a Solar Simulator developed by Photovoltaic Solar Energy Systems Certification Laboratory. These results are used to calculate the errors that determining the classification in accordance with IEC 60904-9. The procedure and points quantity for this kind of analysis is defined by the same standard. However, these procedures would bring errors to estimating the classification in the first calibration procedures according our results. Through several maps with increasing number of measurement points (2 to 1620 ), it was observed in a convergence analysis that it is necessary to increase the number of measurements to find an real erro.
\end{abstract}

Keywords: Solar Simulator, Non-unifotmity, standard. 


\section{INTRODUÇÃO}

Um Simulador Solar é um equipamento utilizado para simular a irradiação solar e serve para realizar diversos estudos em painéis solares dentro de ambientes controlados. Ele pode ser utilizado para duas aplicações diferentes, sendo elas: medição de grandezas elétricas ou exposição da irradiação por longos períodos para avaliar resistência à temperatura. De acordo com a norma IEC 60904-9, o desempenho dos Simuladores Solares é classificado a partir de três níveis, classe A, classe $B$ e classe $C$, sendo a classe A a melhor de todas. Esta classificação é determinada a partir do estudo de três caraterísticas: correspondência espectral, estabilidade temporal e Não-Uniformidade [1], sendo esta última o objeto de análise deste trabalho. Este trabalho visa realizar diversas análises de Não-Uniformidade partindo dos critérios que se encontram especificados na norma antes mencionada. $O$ procedimento indica que deve-se realizar medidas de irradiância em diversos pontos na área de teste utilizando uma célula de silício encapsulada, ou um mini módulo fotovoltaico devidamente calibrado. A área precisa ser dividida em pelo menos 64 pontos equidistantes, ou em subáreas que não ultrapasse os $400 \mathrm{~cm}^{2}$. A obtenção da classificação baseia-se no cálculo do erro utilizando uma equação que relaciona a irradiância máxima e mínima medida nessa área [2].

O Simulador Solar desenvolvido no Laboratório de Certificação de Componentes de Sistemas de Energia Solar Fotovoltaica (LabSolar) possui uma área de teste de $200 \mathrm{~cm} \times 100 \mathrm{~cm} \mathrm{[3]} \mathrm{resultando} \mathrm{em} \mathrm{subáreas} \mathrm{de} 312 \mathrm{~cm}^{2}$ ao dividir por 64 , tornando este critério como mínimo de quantidade de pontos a serem realizados segundo a norma. Entretanto, no processo de calibração, observou-se que está consideração, poderia trazer desvios no cálculo do erro e consequentemente um erro na classificação. Na prática, o procedimento para realizar o mapeamento de NãoUniformidade demanda muito tempo e energia, principalmente em Simuladores Solares Contínuos o que pode resultar em risco de sob exposição ou queimadura se não se possuir equipamentos de proteção adequados.

Alguns grupos de pesquisa desenvolveram técnicas para a obtenção destas medidas de forma rápida utilizando vários sensores pré-localizados com a capacidade de adquirir o erro de Não-Uniformidade em menos de um segundo [4]. Contudo, não possui a capacidade de diminuir ou aumentar a quantidade de medidas a serem realizadas em uma mesma área limitando o estudo. Foi construído um sistema mecatrônico de mapeamento automatizado com movimentos cartesianos que permite definir mediante programação a quantidade de pontos ou subáreas a serem medidos numa área de $220 \mathrm{~cm} \times 130 \mathrm{~cm}$. Ele possui uma célula de silício monocristalino como detector que permite relacionar a posição com um valor de irradiância [5]. A célula foi construída e calibrada segundo a norma IEC 60904-2 [6] utilizando um simulador de bancada AAA.

O objetivo do trabalho é verificar se as condições e procedimentos previstos na norma IEC 60904-9 resulta em resultados que sejam confiáveis na hora da verificação da classificação, se houver ou não, da Não-Uniformidade em equipamentos desenvolvidos experimentalmente, principalmente em etapas de calibração e verificação. A motivação da realização desta análise baseia-se na necessidade de introduzir de maneira objetiva e clara o estudo de Não-Uniformidade de um simulador solar de grande porte, a fim de melhorar a classificação bem como aumentar seu nível 
de eficiência para a caracterização de painéis solares. O LabSolar pretende obter a certificação do INMETRO para empregar exaustivamente este instrumento em diversos testes como: obtenção dos coeficientes de temperatura, curva I-V, hot-spots entre outros.

\section{METODOLOGIA}

A pesquisa realizada foi do tipo experimental, exploratória, teórica e aplicada, já que foi determinado como objeto de estudo e de questionamento, a análise de NãoUniformidade realizada aos simuladores solares para determinar a classificação segundo a norma IEC60904-9, 2007.

\subsection{Simulador Solar}

O Simulador Solar está composto por um sistema de elevação automotivo que define o nível de irradiância ao aproximar ou afastar a fonte de luz do plano de estudo. Esta fonte de luz está composta por 40 holofotes que internamente possuem uma lâmpada de vapor metálico de terras raras especialmente selecionada. Foi estabelecido um distanciamento de trabalho entre os holofotes e o plano de estudo, bem como um mesmo tempo de aquecimento das lâmpadas para atingir uma estabilidade térmica. Esta distância e o tempo foram mantidos para todos os mapeamentos com o objetivo de não modificar a irradiância total, sendo eles de $1 \mathrm{~m}$ e 30 min respetivamente.

\subsection{Mapeador de Não-Uniformidade}

Foi desenvolvido pela equipe do LabSolar e o Laboratorio de Propriedades Óticas (Lapo), um sistema automatizado composto por motores de passo, fusos para locomoção e uma célula calibrada para realizar mapeamentos do plano de estudo. Este sistema é controlado por um microcontrolador $\mathrm{CH} 340$ e dois drivers para motor de passo unipolar completamente desenvolvido pela equipe de trabalho. Ele permite, mediante comandos via serial, definir a quantidade de pontos a serem realizados em uma área determinada.

O sistema de deslocamento cartesiano observado na figura 1 utiliza como parâmetros de entrada (inputs) a quantidade de linhas e colunas que se pretende realizar em uma área determinada, sendo que o deslocamento entre linhas e colunas são calculados automaticamente pelo sistema de controle eletrônico.

Figura 1: Sistema de mapeamento automatizado para determinar a Não-Uniformidade de Simuladores Solares de Grande Área desenvolvido no LabSolar e o Lapo.

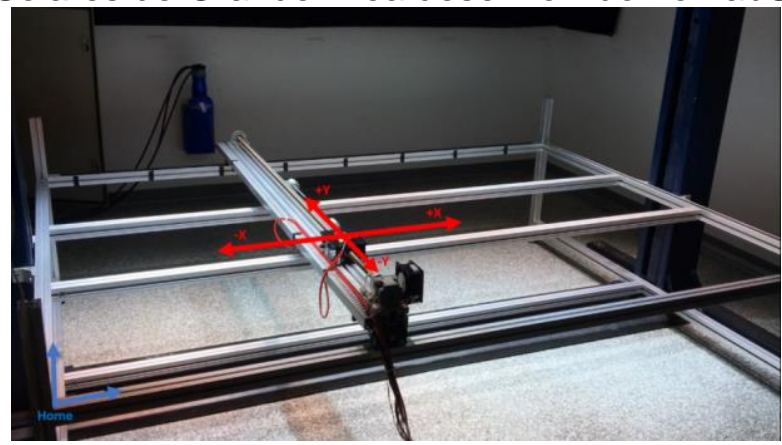


A partir da área de teste, determinamos uma quantidade crescente de medidas a serem realizadas no plano para o mapeamento de Não-Uniformidade. Foi estipulado o número de linhas e colunas (tabela 1), afim de obter as medidas necessárias para calcular a classificação correspondente. O sistema divide automaticamente a área em uma quantidade de subáreas iguais onde o sistema se posiciona e calcula a média entre 100 medidas de irradiância realizadas no local. Evidentemente, ao aumentar o número de sub-áreas, a análise consome um maior tempo tendo uma relação linear com a quantidade de medidas a serem realizada.

Tabela 1: Dados de entrada (Linhas e Colunas) para realização dos mapeamentos de Não-

Uniformidade com o tempo estimado para cada analise.

\begin{tabular}{|c|c|c|c|c|}
\hline Mapa & $\begin{array}{c}\text { Linhas } \\
(\mathbf{L}=\#)\end{array}$ & $\begin{array}{c}\text { Colunas } \\
(\mathbf{C}=\#)\end{array}$ & $\begin{array}{c}\text { Número de sub- } \\
\text { áreas }\end{array}$ & $\begin{array}{c}\text { Tempo estimado } \\
\text { (hh:min) }\end{array}$ \\
\hline $\mathrm{a}$ & 3 & 4 & 12 & $0: 22$ \\
\hline $\mathrm{b}$ & 5 & 6 & 30 & $0: 33$ \\
\hline $\mathrm{c}$ & 6 & 10 & 60 & $0: 55$ \\
\hline $\mathrm{d}$ & 9 & 14 & 126 & $1: 17$ \\
\hline $\mathrm{e}$ & 10 & 16 & 160 & $1: 28$ \\
\hline $\mathrm{f}$ & 11 & 18 & 198 & $1: 39$ \\
\hline $\mathrm{g}$ & 14 & 22 & 308 & $2: 01$ \\
\hline $\mathrm{h}$ & 16 & 26 & 416 & $2: 23$ \\
\hline $\mathrm{i}$ & 21 & 34 & 714 & $3: 07$ \\
\hline $\mathrm{j}$ & 27 & 44 & 1188 & $4: 02$ \\
\hline $\mathrm{k}$ & 41 & 68 & 2788 & $6: 14$ \\
\hline
\end{tabular}

Após inseridos os inputs do sistema, que consiste em dar as coordenadas estipuladas na tabela ( $L$ e $C$ ), procura-se o início de curso de cada eixo e inicia-se o teste utilizando um comando no teclado do computador mediante o monitor serial do sistema.

\subsection{Tratamento dos Dados}

Para os dados que serão obtidos dos mapeamentos anteriores, utilizou-se uma folha de cálculo de Excel para visualização gráfica dos mapas. Foi utilizada a ferramenta de formato condicional que constrói uma escala de cores a partir do valor de cada célula do mapa. Para o máximo valor de irradiância foi adotado o vermelho, para o menor valor a cor verde, e para os valores intermediários uma degradação dessas cores passando pelo amarelo.

Segundo IEC60904-9, o erro associado à Não-Uniformidade é calculado utilizando a equação 1. Nesta análise serão excluídas as bordas dos mapas que em geral estão com valores muito inferiores em relação a média das medidas centrais da área de teste.

$$
E_{\text {Não-Uniformidade }}=\frac{\text { Irra.Máx }- \text { Irra.Mín }}{\text { Irra.Máx }+ \text { Irra.Mín }} * 100 \%
$$




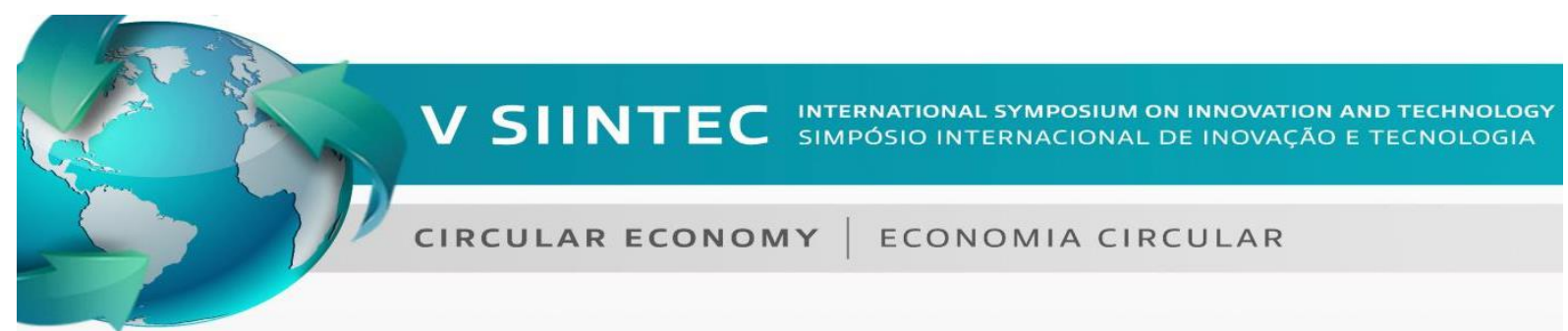

Como resultado final, foi elaborado um gráfico de convergência do erro associado a quantidade de pontos obtidos através do experimento, utilizando-se para este fim, o cálculo do erro por subáreas. A partir do gráfico será possível verificar se há uma tendência dos valores se repetir.

\section{RESULTADOS E DISCUSSÃO (ARIAL 12)}

Com base nos dados obtidos, foram elaborados os mapas de irradiância. A ferramenta de formatação condicional de Excel permitiu observar os valores fora da média central. A figura 2 mostra cada um dos mapas obtidos de acordo a tabela 1.

Figura 2: Mapas de Não-Uniformidade gerados a partir dos dados coletados com o sistema de medição automatizado a partir dos dados da tabela 1.

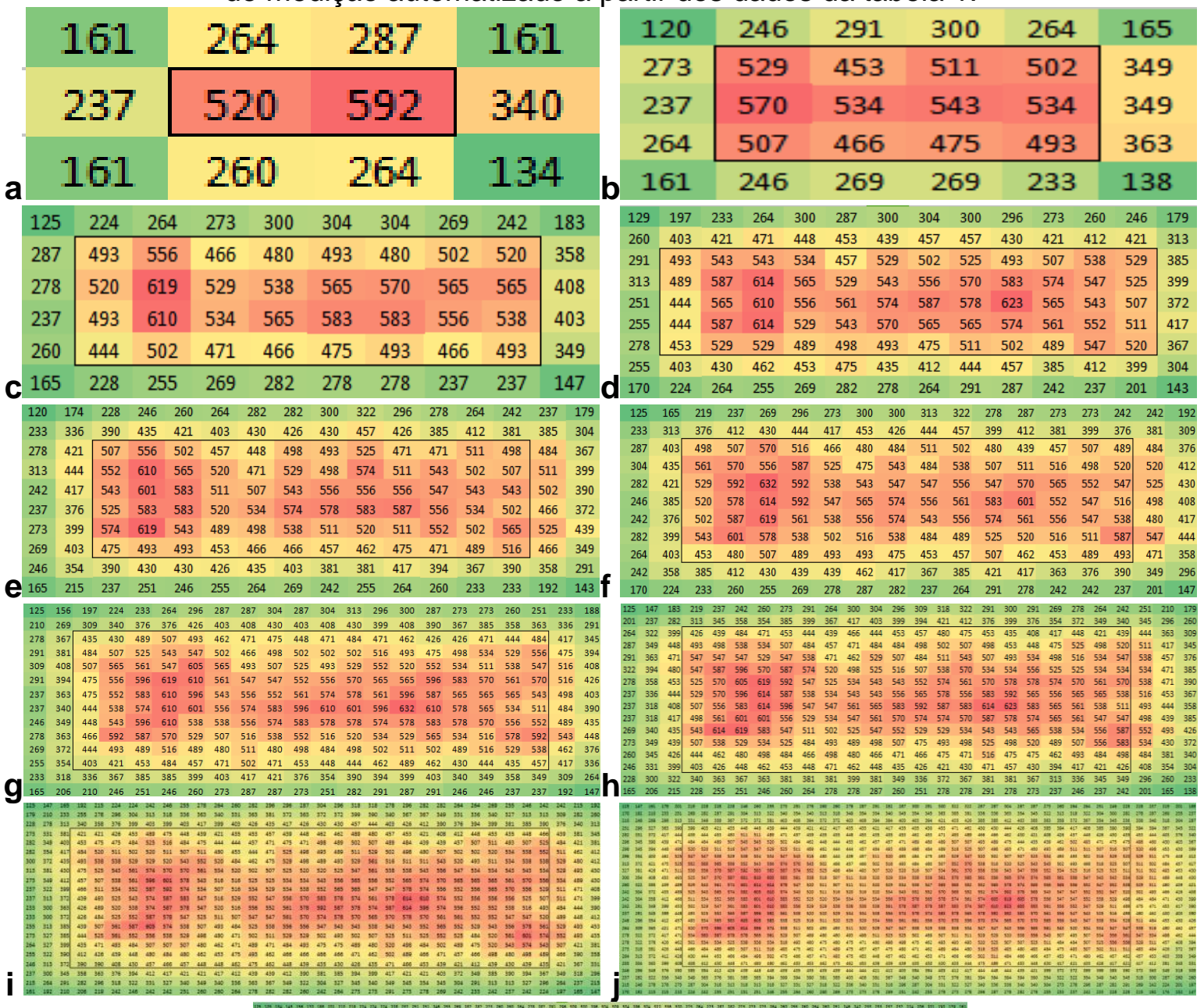

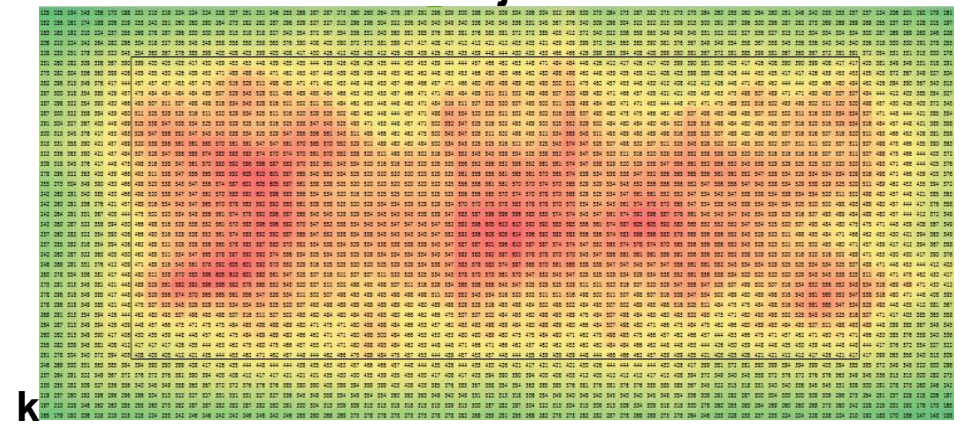


Foram considerados somente a área central dos mapas para calcular a NãoUniformidade. Os valores que se encontram nas bordas estão fora da média dos dados centrais, por exemplo no mapa "c", o total de pontos segundo a tabela 1 é de 60 medidas, mas foram consideradas só as 28 medidas centrais representadas por um retângulo de borda mais escura.

Após o tratamento dos dados para cada quantidade de pontos, existe um valor associado de erro calculado utilizando a equação 1. Estes valores são apresentados na tabela 2 junto com os mínimos e máximos encontrados. Adicionalmente foi calculado a irradiância média (Irra.Méd) que seria a radiação efetiva no plano de teste para essa condição.

Tabela 2: Erro associado à Não-Uniformidade a partir da área interna de cada mapa da fig 2.

\begin{tabular}{|c|c|c|c|c|c|c|c|}
\hline Mapa & L,int. & C,int. & $\begin{array}{c}\text { Pontos } \\
\text { int. }\end{array}$ & $\begin{array}{c}\text { Irra.Máx. } \\
\left(\mathbf{W} / \mathbf{m}^{\mathbf{2}} \mathbf{)}\right.\end{array}$ & $\begin{array}{l}\text { Irra.Mín } \\
\left(\mathbf{W} / \mathbf{m}^{\mathbf{2}} \mathbf{)}\right.\end{array}$ & $\begin{array}{c}\text { Irra.Méd } \\
\left(\mathbf{W} / \mathbf{m}^{\mathbf{2}} \mathbf{)}\right.\end{array}$ & $\begin{array}{c}\mathbf{E}_{\text {Não-Uniformidade }} \\
(\mathbf{\%})\end{array}$ \\
\hline $\mathrm{a}$ & 1 & 2 & 2 & 592,0 & 520,1 & 556,0 & 6,46 \\
\hline $\mathrm{b}$ & 3 & 4 & 12 & 569,5 & 452,7 & 509,6 & 11,42 \\
\hline $\mathrm{c}$ & 4 & 7 & 28 & 618,9 & 443,7 & 522,9 & 16,49 \\
\hline $\mathrm{d}$ & 6 & 11 & 66 & 623,4 & 443,7 & 537,2 & 16,84 \\
\hline $\mathrm{e}$ & 7 & 12 & 84 & 618,9 & 448,2 & 523,6 & 16,00 \\
\hline $\mathrm{f}$ & 8 & 13 & 104 & 632,4 & 439,2 & 530,8 & 18,02 \\
\hline $\mathrm{g}$ & 10 & 17 & 170 & 632,4 & 403,3 & 527,1 & 22,12 \\
\hline $\mathrm{h}$ & 12 & 19 & 228 & 623,4 & 394,3 & 520,2 & 22,51 \\
\hline $\mathrm{i}$ & 15 & 27 & 405 & 614,4 & 389,8 & 513,4 & 22,36 \\
\hline $\mathrm{j}$ & 20 & 34 & 680 & 623,4 & 385,3 & 519,4 & 23,60 \\
\hline k & 30 & 54 & 1620 & 614,4 & 380,9 & 513,2 & 23,47 \\
\hline
\end{tabular}

Com base nestes valores foi gerado o gráfico de convergência. Figura 3, que representa a partir de que quantidade de pontos medidos, o erro associado tende a um valor representativo. Observou-se que a partir de 170, o erro não variou significativamente obtendo um valor médio de $22,81 \%$.

Figura 3: Gráfico de convergência do erro de Não-Uniformidade em função do número de pontos internos considerados em cada mapa realizado.

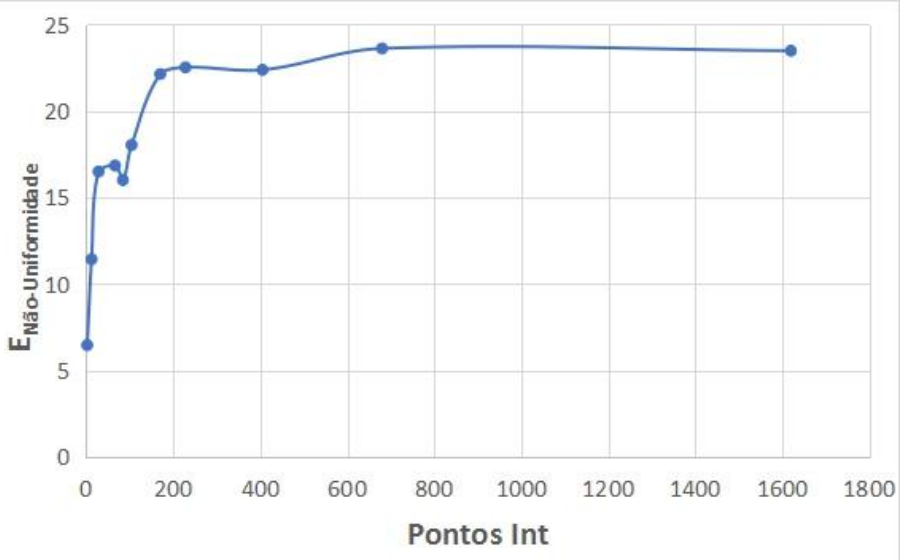




\section{CONCLUSÃO (ARIAL 12)}

A norma IEC 60904-9 estabelece que a quantidade de pontos necessários para realizar os testes de Não-Uniformidade deve atender o mínimo de 64 pontos dentro da área de teste. A partir das análises efetuadas ao decorrer deste trabalho constatouse que o valor estipulado pela norma não é suficiente para simuladores que estão em período de calibração, pois resulta em um erro longe do valor real, sendo que para o estado atual precisa-se no mínimo 170 pontos para aproximar-se a um erro mais exato. Sugere-se que, para se definir este número de pontos a serem realizados, deve-se levar em conta a análise de convergência onde se evidencia o comportamento real do erro em função do número de medidas realizadas.

Os sistemas que já se encontram na literatura não possuem a capacidade para a realização da análise proposta. A capacidade do nosso sistema de medição com posicionamento cartesiano automático, facilita a obtenção destas medidas ao serem totalmente customizável.

Apesar dos resultados favoráveis que foram obtidos o Simulador ainda não possui classificação já que o erro é maior a 10\%. Contudo ainda estamos em fase de aperfeiçoamento com o intuito de obter uma classificação e consequentemente uma certificação.

\section{Agradecimentos}

Inicialmente o meu agradecimento é a Deus, o autor e consumador da fé.

As equipes do LabSolar e o Lapo que participaram no desenvolvimento deste trabalho.
A COELBA e a SECTI pela construção e manutenção do Labsolar.
E CAPES e CNPq pelo apoio financeiro.

\section{REFERÊNCIAS (ARIAL 12)}

${ }^{1}$ COSTA E., OLIVEIRA F., KRENZINGER A., Desenvolvimento de simulador solar para exposição continua de módulos fotovoltaicos. VII Congresso Brasileiro de Energia Solar - Gramado, 2018.

\section{${ }^{2}$ Norma IEC60904 - 9 : 2007. Photovoltaic devices - Part 9: Solar simulator performance requirements.}

${ }^{3}$ Alfonzo J., Paes T., Pepe I., López-Agüera A. Selection of Light Source Solar Simulator for different Spectral Distribution Research. Int. Journal for Research in Applied Science \& Eng. Tech. V 6, Issue XII, 2018.

${ }^{4}$ Rivola D., Dittmann S., Pravettoni M., Friesen G., Chianese D., High-Speed MultiChannel System for Solar Simulator Irradiance Non-Uniformity Measurement. Conference: 40th IEEE Photovoltaic Specialists Conference.

${ }^{5}$ Norma IEC60904 - 2 : 2007. Photovoltaic devices - Part 2: Requirements for photovoltaic reference devices. 
${ }^{6}$ Alfonzo J., Achy A., Paes T., Pepe I. Medidor de no uniformidad para caracterizar simuladores solares de estado estable de gran superficie. VII Congresso Brasileiro de Energia Solar, Gramado 2018. 